\title{
Características sociodemográficas e avaliação da dor através do questionário de McGill em mulheres com dismenorreia primária submetidas à auriculoterapia
}

\author{
Sociodemographic characteristics and pain \\ assessment through the McGill questionnaire in \\ women with primary dysmenorrhoea submitted to \\ auriculotherapy
}

\author{
Fernanda Ferreira de Sousa' ${ }^{1}$ \\ José Francisco Miranda de Sousa Junior ${ }^{2}$ (1) \\ Patrícia Lima Ventura ${ }^{3}$ (1)
}

\begin{abstract}
1'Autora para correspondência. Centro Universitário Santo Agostinho (Teresina). Piauí, Brasil. fernandasousafsa@gmail.com
\end{abstract}
${ }^{2,3}$ Centro Universitário Santo Agostinho (Teresina). Piauí, Brasil. fisiojuniormiranda@gmail.com, vlpatricia@hotmail.com

\begin{abstract}
RESUMO | OBJETIVO: A dismenorreia caracteriza-se como uma dor intensa durante a menstruação ou algumas horas antes, localizada na região de baixo ventre ou região inferior do abdômen, é uma patologia de origem primária ou secundária, em que 40 a $45 \%$ dos casos de dor pélvica crônica, se manifestam como dismenorreia. OBJETIVO: Investigar as características sociodemográficas de mulheres que possuem dismenorreia primaria, e avaliar as influências da auriculoterapia para a dor menstrual, mensurada antes e após o método por meio do questionário de McGill. MÉTODOS: Trata-se de estudo clínico, randomizado, controlado e duplo cego, de delineamento longitudinal, com abordagem quali-quantitativa, realizado por meio de uma população de 118 estudantes. $O$ tamanho da amostra foi calculado usando uma única fórmula média onde $\mathrm{n}$ é o tamanho da amostra necessário; $\mathrm{d}$ é erro marginal de $5 \%$ ( $d=0,05$ ); z é o grau de precisão requerido a $95 \%$ de nível de confiança. Inicialmente, as voluntárias foram submetidas a responderem o questionário sociodemografico, e após, ao questionário de McGill, que avalia vários aspectos da dor, e receberam aplicações durante 2 meses e 3 semanas, 2 vezes por semanas, por 20 minutos. RESULTADOS: A média de idade das participantes desta pesquisa é de 19,8 anos, com predominância na etnia parda e com ciclo menstrual regular, e 55\% apresenta dor dois dias antes da menstruação. Depois das aplicações de auriculoterapia os dados dos questionários de McGill após intervenção apresentaram valores estatísticos significantes nos domínio de aspectos sensitivos, afetivos, domínios avaliativos e aspectos miscelâneos. CONCLUSÃo: O presente estudo obteve o delineamento nos aspectos sociodemograficos da população que possui dismenorreia primária. E em relação às influências da auriculoterapia sobre as características da dor, o método apresentou se estatisticamente significativo.
\end{abstract}

PALAVRAS-CHAVE: Dismenorreia. Dismenorreia primária. Acupuntura. Auriculoterapia.
ABSTRACT | OBJECTIVE: Dysmenorrhea is characterized as severe pain during menstruation or a few hours before, located in the lower abdomen or lower abdomen, it is pathology of primary or secondary origin, in which 40 to $45 \%$ of pain cases chronic pelvic, manifests as dysmenorrhea. OBJECTIVE: To investigate the sociodemographic characteristics of women who have primary dysmenorrhea, and to evaluate the influence of auriculotherapy for menstruation, measured before and after the McGill questionnaire method. METHODS: This is a clinical, randomized, controlled and double-blind study, with a longitudinal design, with a qualitative and quantitative approach, carried out with a population of 118 students. The sample size was calculated using a single average formula, where the sample size is required; marginal error of $5 \%(d=0.05)$; $z$ is the degree of precision required at $95 \%$ of the confidence level. Initially, as volunteers, they were submitted to a sociodemographic questionnaire, and then to McGill's questionnaire, which evaluated various aspects of pain, and applied it for 2 months and 3 weeks, twice a week, for 20 minutes. RESULTS: The average age of the participants in this research is 19.8 years, with a predominance of mixed race and with regular menstrual cycle, and $55 \%$ present pain two days before menstruation. After auriculotherapy applications and data from McGill's questionnaires, after the intervention of significant statistical values in the domain of sensitive, affective, assessed domains and diverse aspects. CONCLUSION: The present study used or the design in the sociodemographic aspects of the population that has primary desmenorrhea. And in relation to the influences of auriculotherapy on the characteristics of pain, the method presented is statistically significant.

KEYWORDS: Dysmenorrhea. Primary Dysmenorrhea. Acupuncture. Auriculotherapy. 


\section{Introdução}

A dismenorreia caracteriza-se como uma dor intensa durante a menstruação ou algumas horas antes, localizada na região de baixo ventre ou região inferior do abdômen, é uma patologia de origem primária ou secundária, em que $40 \%$ a $45 \%$ dos casos de dor pélvica crônica (DPC) se manifestam como dismenorreia. A dismenorreia primaria (DP) é definida como dor menstrual com ausência de lesões orgânicas é de etiologia não totalmente definida, no meio científico é descrito que, durante o fluxo menstrual há uma maior liberação de prostaglandinas, o que causa maior contração uterina e dor. Cerca de $80 \%$ das mulheres nulíparas possui a $\mathrm{DP}^{1,2,5}$.

São fatores agravantes da DP, uma menarca precoce, massa corporal baixa ou alta, fluxo menstrual prolongado ou aberrante, histórico familiar de dismenorreia, tabagismo, fatores nutricionais como a ingestão excessiva de cafeína. Essa condição ginecológica é mais prevalente em mulheres em idade reproduti$\mathrm{va}^{3,4}$, com início na adolescência após o estabelecimento dos ciclos ovulatórios. A prevalência geral entre adolescentes com DP é de $60 \%$ a $90 \%$, e diminui com o aumento da idade ${ }^{5,6,7}$.

A dismenorreia secundaria (DS) é decorrente de processos orgânicos, que causa congestão pélvica ou espasmo uterino. Dentre esses estão à endometriose, leiomioma, prolapsos pélvicos, má formação genital, estenose do canal do colo do útero e outras afecções. Na DS as prostaglandinas também estão presentes, porém os fatores desencadeantes da dor são anatômicos, e a liberação de prostaglandinas está interligada as sintomatologias de base $e^{1,9,10}$.

As sintomatologias frequentes que acompanham a dismenorreia são as dores lombares, náuseas, vômitos e diarreia, que resultam negativamente na qualidade de vida e favorece na ausência de curto prazo na escola ou no trabalho de acordo com o nível de gravidade ${ }^{7,8}$. Atualmente a diversos tratamentos para da dismenorreia, tanto por meios farmacológicos através do uso de anti-inflamatórios não esteroides (AINEs), como não farmacológicos ${ }^{11}$.
O tratamento sintomático visa remover ou aliviar a dor. Na fase de profilaxia podem ser adotadas medidas cirúrgicas, terapias alternativas, mudanças no estilo de vida como adesão a práticas de atividade física e na alimentação $0^{1,11,12}$. Dentre os tratamentos não farmacológicos, a medicina tradicional chinesa é uma forma de método alternativo para diversos acometimentos em relação à saúde, e dentro dela à acupuntura que em vários estudos têm se mostrado com efeitos terapêuticos positivos na $\mathrm{DP}^{13}$.

Dentro da acupuntura existe à auriculoterapia que utiliza agulhas, sementes ou cristais, na qual são realizadas aplicações em pontos específicos da orelha. A estimulação desses pontos transmite sinais para o cérebro e a órgãos específicos modulando e harmonizando as suas funções fisiológicas. O pavilhão auricular apresenta zonas reflexas, é denominado como um microssistema com a representação de todos os órgãos e estruturas do corpo humano ${ }^{14}$. Objetivo deste estudo é Investigar as características sociodemográficas de mulheres que possuem dismenorreia primaria, e avaliar as influências da auriculoterapia para a dor menstrual, mensurada antes e após o método por meio do questionário de McGill.

\section{Métodos}

Este estudo é um ensaio clínico randomizado e duplo cego, com abordagem quantitativa e qualitativa, aprovado pelo comitê de ética em pesquisa (CAAE: 80289517.0.0000.5602) através da Plataforma Brasil com o Parecer: 2.423.373. Está pesquisa foi realizada em uma instituição de ensino superior, no período de março a junho de 2018. Por meio de uma população de origem de 168 alunas de um curso superior, ocorreu uma subtração aleatória simples de 118 estudantes (população estudada). 
O tamanho da amostra foi calculado usando uma única fórmula média, onde $\mathrm{n}$ é o tamanho da amostra necessário; $d$ é erro marginal de $5 \%(d=0,05)$; z é o grau de precisão requerido a $95 \%$ de nível de confiança. Após o cálculo amostral a população de origem foi selecionada através da lista de frequência do $2^{\circ}$ ao $8^{\circ}$ período.

Através da lista de frequência, foi contabilizada 168 frequentantes, logo após aplicou se o calculo amostral, onde a amostragem com significância era de 118 alunas, estas foram selecionadas através da forma aleatória simples, onde seus números correspondentes à lista de chamada foram colocados em sorteios numéricos em uma urna preta.

Em seguida as participantes selecionadas assinaram o Termo de consentimento livre e esclarecido (TCLE), e responderam ao questionário sociodemografico (QS) que foi desenhado pelos autores da pesquisa de acordo com as características da DP, conforme o Guideline de DP. Composto por 20 questões que abordava os seguintes aspectos: curso; período; profissão; etnia; como é o ciclo menstrual, regular com duração de 27 a 32 dias para iniciar um novo ciclo ou irregular com duração menor ou maior que 27 a 32 dias para iniciar um novo ciclo; quantos dias duravam a menstruação; se surgirão dores dois dias antes da menstruação; após três dias de menstruação as dores desaparecem, diminuíam ou continuavam; durante o período menstrual essas dores se espalhavam por outras regiões do corpo. Se sim quais.

Quais sintomas apresentavam durante a menstruação; possuía histórico familiar de dor de cólica; estava fazendo uso de algum método contraceptivo; quando estava menstruada faltava ao trabalho ou a faculdade; fazia exercício físico regularmente; quais exercícios estavam praticando atualmente; fazia uso de algum tratamento medicamentoso; Possuía histórico de alguma cirurgia abdominal ou pélvica; estava fazendo alguma terapia; histórico médico de patologias como endometriose, fibroma ou outra patologia pélvica; possuía histórico de parto ou aborto; além da menstruação possuía alguma outra doença que the causasse dor; fumante; Possuí fobia de agulhas.
Após a aplicação do (QS), das 118 mulheres permaneceram na pesquisa as que possuíam os seguintes critérios de inclusão, idade entre 18 a 25 anos com diagnóstico provável de dismenorreia primária e ciclo menstrual regular entre 27 a 32 dias, sedentárias, não tabagistas, não praticantes de métodos de tratamento medicamentoso ou fisioterapeuticos, está cursando entre $\circ 2^{\circ}$ a $8^{\circ}$ período do curso superior sorteado para a pesquisa.

Os critérios de exclusão foram atribuídos para, alunas com características de dismenorreia secundária, doenças físicas que causam dor, gravidez ou histórico de parto, fazer uso de qualquer droga psicotrópica e contracepção hormonal nos últimos seis meses, histórico de doenças cardíacas, participantes com fobias de agulhas, e que não responderam ao TCLE e ao questionário sociodemográfico por completo.

A população do estudo foi constituída por $118 \mathrm{mu}$ Iheres com DP, sendo que 97 participantes, por não estarem adequadas aos critérios de elegibilidade, tais como: histórico de gravidez e/ou doenças base associadas $(n=37)$, uso de drogas psicotrópicas ou contracepção hormonais $(n=35)$ e participantes sem disponibilidade de tempo para realizar as intervenções, praticantes de atividade física ou outros tratamentos fisioterapeuticos ou e faltaram na fase triagem inicial do estudo $(n=25)$.

Após a avaliação pelos critérios de inclusão e exclusão, foram incluídas 21 voluntárias, que foram divididas de forma aleatória simples nos grupos controlado e experimental $A$, e controlado e experimental $B$, totalizando quatro grupos. Após a randomização simples os grupos controlados A, B e experimental B ficaram com cinco participantes cada, e o controlado B com seis.

O questionário de McGill foi utilizado para avaliar antes e após a intervenção os aspectos da dor com a ajuda de palavras escolhidas pelos participantes, como forma de expressar a dor que é sentida, sendo esses descritores divididos em quatro grupos de questões: sensorial-discriminativo (subgrupo de 1 a 10), afetivo-motivacional (subgrupo de 11 a 15), avaliativo-cognitivo (subgrupo 16), e miscelânea (subgrupo de 17 a 20). 
Foram montados dois protocolos diferentes. O primeiro é composto pelos pontos auriculares. Simpático: Rim: Shen Men "porta da alma": Ovário: Útero: Endócrino. O segundo protocolo de intervenção e formado pelos mesmos pontos citados acima exceto o ponto Simpático que foi substituído pelo ponto fígado. Os protocolos também foram separados de forma aleatória para os grupos em sorteados em duas urnas pretas, uma com os pares dos grupos, experimental e controle A e experimental e controle $B$, na outra urna foram depositado os protocolos.

O primeiro protocolo contendo o ponto simpático e os demais pontos foi designado aos grupos controlados A e experimental A, e o segundo protocolo contendo o ponto fígado ficou para o controlado B e experimental B. As 21 voluntárias foram submetidas à randomização, por meio do software online gratuito (www.random.org), as quais foram organizadas em quatro grupos distintos: grupo controle A - (simpático) $(n=5)(G C-A)$, grupo experimental $A$ - (simpático) $(n=5)(G E-A)$, grupo controle $B-$ (fígado) $(n=6)(G C-B)$ e grupo experimental $B$ - (fígado) $(n=5)(G E-B)$.

As aplicações foram realizadas no período de 2 messes e 3 semanas, distribuídas em 2 vezes por semana com duração de 20 minutos, totalizando 22 intervenções, que compreenderam 3 ciclos menstruais. As voluntárias foram posicionadas sentadas, com o intuito da realização da inspeção auricular, em segui$\mathrm{da}$, foram executados os procedimentos de assepsia no pavilhão auricular, através do uso de algodão com álcool a 70\%, em seguida, foram inseridas agulhas estéreis, de aço inoxidável, com tamanho padrão $(0.25 \mathrm{x}$ $0.15 \mathrm{~mm}$ ), unilateralmente na orelha direita com tubo de guiamento nos pontos de indicação de acordo com o protocolo de cada grupo experimental. A oreIha direita foi escolhida para os dois protocolos, pois só nela é encontrado o ponto fígado.

Nos grupos controle foi colocado micropore em cada ponto específico correspondente seu protocolo, simulando a aplicação da auriculoterapia. Dois pesquisadores fizeram as intervenções, cada um ficou responsável por dois grupos do início ao fim deste estudo, seguindo sempre a mesma ordem de alocação dos participantes e horários, como também da colocação das agulhas nos pontos auriculares.

Os dados foram analisados com o auxílio do programa Graphpad Prism v. 5. Para análise da distribuição dos dados amostrais foi aplicado o teste de normalidade de Shapiro-Wilk, seguido do teste não paramétrico de Wilcoxon para a comparação intragrupos (antes e depois do tratamento). Na avaliação intergrupo foi executado o teste de Kruskal-Wallis com pós-teste de Dunn's. Para todos os testes foi considerando um nível de significância de $5 \%(p<0,05)$.

\section{Resultados}

Seleção amostral do estudo após os critérios de inclusão e exclusão. A amostra final desta pesquisa foi composta por 21 participantes. 
Figura 1. Fluxograma da seleção amostral para o estudo.

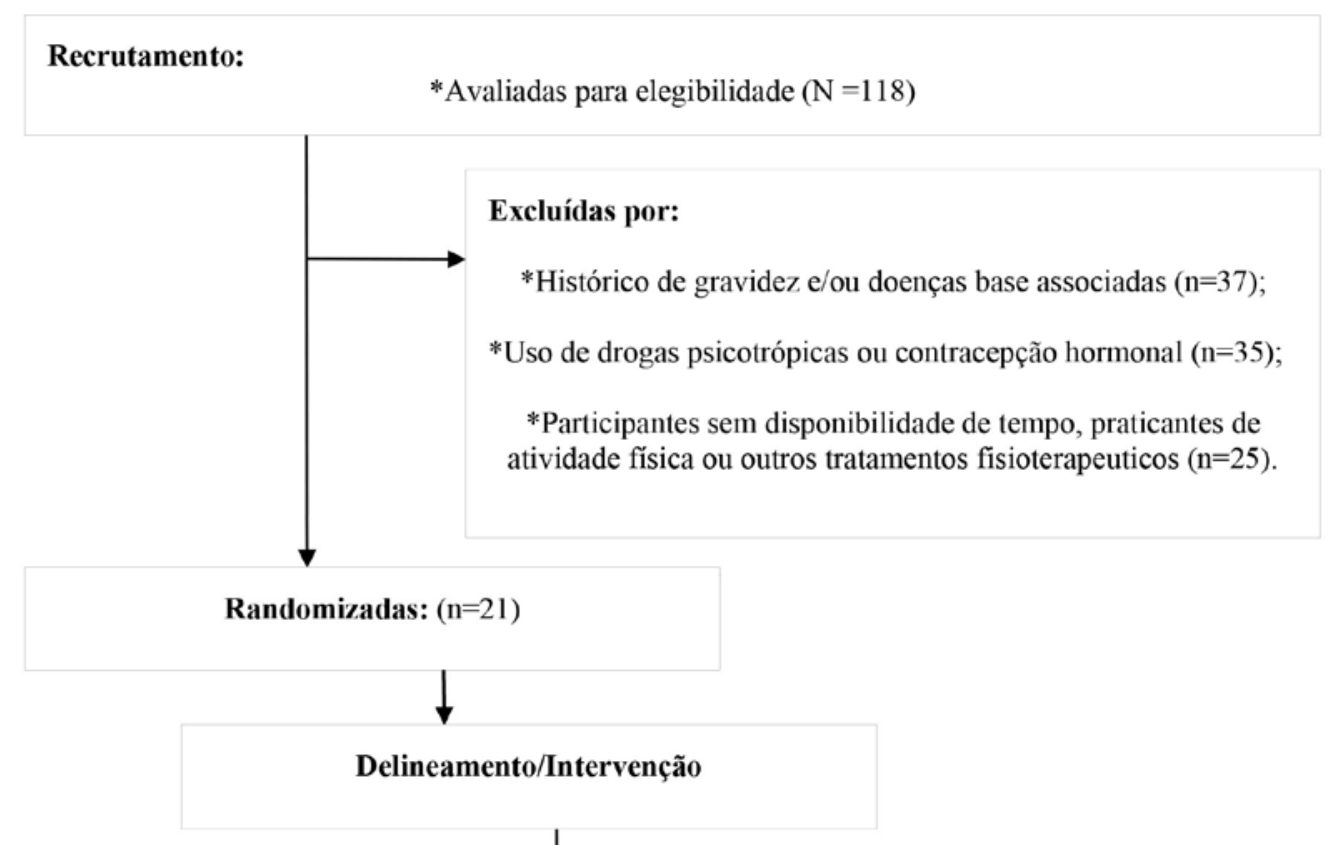

Grupo experimental A (simpático) - aplicação das agulhas seguintes acupontos (simpático, rim, shen men, ovário, útero c endócrino).

Grupo experimental B (fígado) - aplicação das agulhas seguintes acupontos (figado, rim, shen men, ovário, útero e endócrino).

O protocolo foi conduzido por 2 meses e 3 semanas, sendo 2 vezes por semana, por 20 minutos, totalizando 22 intervenções.
Grupo controlado A (simpático) - aplicação do micropore nos seguintes acupontos (simpático, rim, shen men, ovário, útero e endócrino).

Grupo controlado B (fígado) - estimulação dos seguintes acupontos (figado, rim, shen men, ovário, útero e endócrino).

O protocolo foi conduzido por 2 meses e 3 semanas, sendo 2 vezes por semana, por 20 minutos, totalizando 22 intervenções.

\section{Acompanhamento}

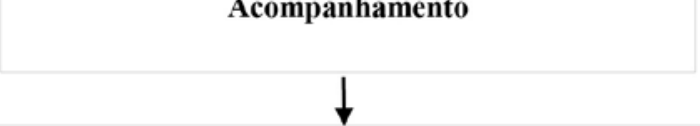

Grupo experimental A (simpático): $(\mathrm{n}=5)$ Grupo controle A (simpático): $(n=5)$
Grupo experimental B (figado): $(n=5)$

Grupo controle B (fígado): $(\mathrm{n}=6)$

\section{Perda Amostral}

*Voluntários que não foram acompanhados até o fim do estudo $(\mathrm{n}=0)$

*Participantes que tiveram a intervenção interrompida $(\mathrm{n}=0)$

*Voluntários que não realizaram a rotina de treinos até o fim do estudo $(\mathrm{n}=0)$

*Participantes que interromperam a intervenção $(n=0)$

\begin{tabular}{c}
$\downarrow$ \\
Amostra Final: \\
$(\mathrm{n}=21)$ \\
\hline
\end{tabular}


A caracterização amostral dominante estabelecida segundo o questionário sociodemografico: Na tabela 1 a média de idade das participantes desta pesquisa é de 19,8 anos, com predominância na etnia parda e com ciclo menstrual regular. E 57\% apresentam dor dois dias antes da menstruação, a região lombar e o local mais acometido por sintomas álgicos no período menstrual acometendo $43 \%$ das participantes.

Durante o período menstrual a dor de cabeça e irritação nervosa atinge $23 \%$ das participantes e $27 \%$ não manifesta nenhum sintoma aparente. A sudorese associada à dor menstrual é o sintoma de maior predominância afetando 45\%, a maioria desconhece histórico familiar de dismenorreia, 50\% histórico materno e 64\% fraterno. O único método contraceptivo utilizado pelas participantes selecionadas nesta pesquisa foi à camisinha cerca de $10 \%$. No Período menstrual $32 \%$ às vezes acontecem absenteísmo as aulas e/ ou trabalho no período menstrual.

Tabela 1. Caracterização amostral sociodemográfica das participantes com dismenorreia primária. Teresina, 2018 (continua)

\begin{tabular}{|c|c|c|c|}
\hline \multicolumn{2}{|c|}{ Variáveis } & \multirow{2}{*}{$\begin{array}{l}\text { n } \\
5\end{array}$} & \multirow{2}{*}{$\begin{array}{l}\% \\
24 \%\end{array}$} \\
\hline Idade & 19 & & \\
\hline & 20 & 4 & $19 \%$ \\
\hline & 21 & 7 & $33 \%$ \\
\hline & 22 & 3 & $14 \%$ \\
\hline & 23 & 1 & $5 \%$ \\
\hline & 24 & 1 & $5 \%$ \\
\hline \multirow[t]{5}{*}{ Etnia } & Branca & 2 & $9.5 \%$ \\
\hline & Parda & 16 & 76. $2 \%$ \\
\hline & Negra & 3 & $14.3 \%$ \\
\hline & Amarelo & 0 & $0 \%$ \\
\hline & Indígena & 0 & $0 \%$ \\
\hline
\end{tabular}

Tipo de ciclos

Regular

$100 \%$

Irregular

$\begin{array}{ll}21 & 100 \% \\ 0 & 0 \%\end{array}$

Dores dois dias antes da menstruação

Sim $12 \quad 57 \%$

Após três dias de menstruação as dores

$\begin{array}{lll}\text { Desaparecem } & 18 & 85.7 \% \\ \text { Diminuem } & 3 & 14.3 \% \\ \text { Continuam } & 0 & 0 \%\end{array}$

Dores se espalham para outras regiões do corpo

$\begin{array}{lll}\text { Não } & 8 & 36 \% \\ \text { Região lombar } & 10 & 45 \% \\ \text { Sacro } & 5 & 23 \% \\ \text { Anus } & 1 & 5 \% \\ \text { Região interna das coxas } & 2 & 9 \%\end{array}$




\begin{tabular}{|c|c|c|}
\hline Variáveis & $\mathbf{n}$ & $\%$ \\
\hline \multicolumn{3}{|l|}{ Apresenta no período menstrual } \\
\hline Náuseas & 2 & $9 \%$ \\
\hline Vômitos & 3 & $14 \%$ \\
\hline Diarreia & 3 & $14 \%$ \\
\hline Dor de cabeça & 5 & $23 \%$ \\
\hline Irritação nervosa & 5 & $23 \%$ \\
\hline Prisão de vente & 3 & $14 \%$ \\
\hline Nenhum dos sintomas & 6 & $27 \%$ \\
\hline Outros sintomas & 2 & $9 \%$ \\
\hline \multicolumn{3}{|l|}{ Apresenta quando sente dores } \\
\hline Palidez & 6 & $27 \%$ \\
\hline Sudorese & 10 & $45 \%$ \\
\hline Nenhum dos sintomas & 9 & $41 \%$ \\
\hline \multicolumn{3}{|l|}{ Menstruação dolorosa da mãe } \\
\hline Sim & 8 & $38.1 \%$ \\
\hline Não & 11 & $52.4 \%$ \\
\hline Não declarado & 2 & $9.5 \%$ \\
\hline \multicolumn{3}{|l|}{ Menstruação dolorosa da irmã } \\
\hline Sim & 6 & $28.6 \%$ \\
\hline Não & 14 & $66.7 \%$ \\
\hline Não declarado & 1 & $4.7 \%$ \\
\hline \multicolumn{3}{|l|}{ Uso de métodos contraceptivos } \\
\hline Sim (camisinha) & 2 & $10 \%$ \\
\hline Não & 29 & $90 \%$ \\
\hline \multicolumn{3}{|c|}{ Absenteísmo escolar ou trabalho no período menstrual } \\
\hline Sempre & 0 & $0 \%$ \\
\hline As vezes & 7 & $33.3 \%$ \\
\hline Nunca falta & 14 & $66.7 \%$ \\
\hline
\end{tabular}


A figura 2 apresenta a análise intragrupos e intergrupos dos domínios do questionário de McGill. Nos dados intragrupos, referentes ao domínio de aspectos sensitivos, o GC-A em seus valores iniciais e finais apresentou valores significativos ( $p=0,033)$, enquanto que os (GE- $A$ e GE-B), assim como, o (GC-B), demonstraram valores extremamente significativos $(p=0,0001)$.

$\mathrm{Na}$ analise intergrupos foi verificado que na comparação entre os (GC-A x GE-A) e (GC-A x GE-B) foram evidenciados dados extremamente significativos $(p=0,0001)$. Em relação aos valores correspondentes a variável afetiva, 0 GE-A e GC-B, apresentaram valores extremamente significativos sendo $(p=0,0003)$ e $(p=0,0001)$, respectivamente. Já na análise intergrupos foi verificado que na comparação entre os grupos (GC-A x GE-A) e (GC-A x GE-B) foram dados extremamente significativos $(p=0,0001)$.

Nos domínios avaliativos, na avaliação intragrupos, os grupos: (GE - A) ( $p=0,048)$, e (GC-B) $(p=0,048)$, apresentaram valores significativos, enquanto que na análise intergrupos não houve nenhuma diferença estatística entre os grupos estudados. Os aspectos miscelâneos nos grupos (GE-A e GC- B) se mantiveram com resultados extremamente significativos ( $p=0,0003)$, na análise intergrupos a comparação entre os grupos (GC-A x GE-A) e (GC-A x $G E-B)$ apresenta valores extremamente significativos e significativos, sendo $(p=0,0001)$ e $(p=0 ; 05)$.

Figura 2. Análise das características da dor intra e intergrupos por meio do questionário de McGill
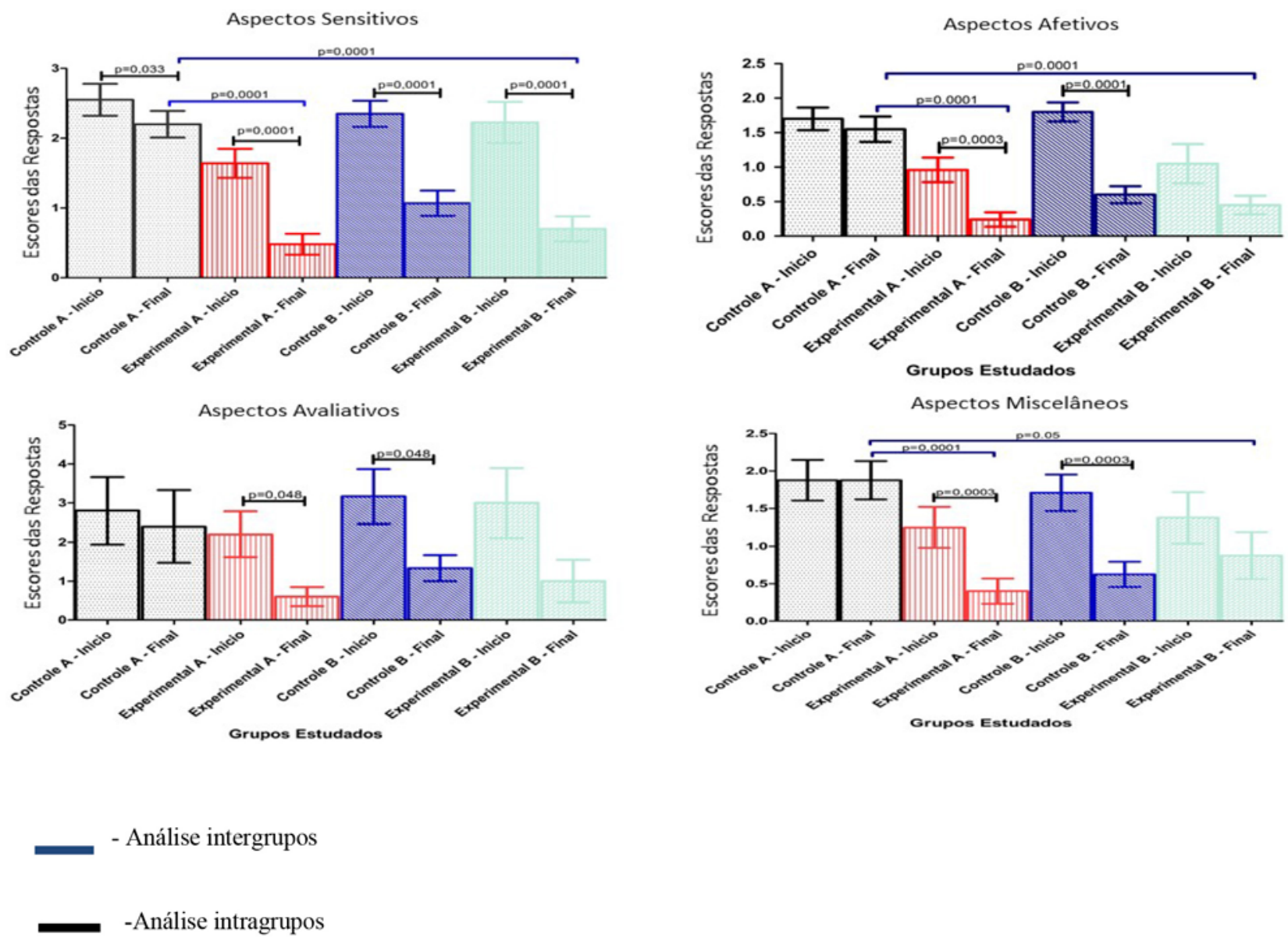

Fonte: Pesquisadores responsáveis, 2018. 


\section{Discussão}

A população desta pesquisa possui idade média de 19,8 anos, predominância da cor parda, todas com ciclo menstrual regular, onde as dores menstruais da maioria apresentam-se dois dias antes da menstruação, e após três dias essas dores desaparecem em $82 \%$ dos casos. Em uma pesquisa realizada com 107 mulheres, a DP surgiu após dois anos da menarca e se manifestava nos dois primeiros dias do fluxo menstrual ${ }^{15}$. O que corresponde aos mesmos dados encontrados nesta pesquisa. Os locais que apresentam maior acometimento de dores associada à menorreia é a região lombar e sacral, de menor ocorrência na região anal e parte interna das coxas.

A prevalência de DP e em $71 \%$ das mulheres com idade média de 20,8 anos, com relação à menarca, em um mesmo estudo e de 12,37 anos, similar a outras pesquisas encontradas com valores de aproximadamente 12 anos $^{16,17}$. Em um estudo randomizado controlado com placebo duplo-cego, em 54 estudantes de ensino superior que apresentavam DP, receberam intervenções do método homeopático, a idade média das participantes era de 22,3 anos, e idade média da menarca de 13,0 anos ${ }^{18}$.

Os sintomas mais comuns durante o período menstrual apontados nesta pesquisa são dores de cabeça, irritação nervosa, vômitos, diarreia e sudorese, 33.3\% da população deste estudo, às vezes têm absenteísmo escolar e no trabalho durante a menstruação. Em uma pesquisa na qual foi investigada as características da dor, nessa as principais queixas apontadas entre as mulheres com dismenorreia é a dor de cabeça, diarréia, náuseas e vômitos. Indicando que os sintomas podem ser antes da menstruação ou no decorrer dos dias menstruais. A dor pode irradiar para a coxa ou a parte inferior das costas. A dismenorreia pode ser descrita como cólica, e a forma com que a dor se apresenta varia entre as mulheres, aquelas com características de dor severa apresentam maiores efeitos negativos ${ }^{19}$.

As características da dor são determinadas através de uma percepção individual, portanto, a dismenorreia, apesar de ser um acometimento global, tem uma concepção individual pelo fato da natureza da dor ser pessoal ${ }^{19}$. Um estudo exploratório recrutou um grupo de 106 voluntárias que eram representativos de mulheres na comunidade com dismenorreia, com vários sintomas incluindo dor nas costas, fadiga, sensibilidade mamária, náuseas, dores de cabeça e alterações intestinais, consistente com muitas outras populações com dismenorreia primária ${ }^{20}$.

Em mulheres com histórico familiar de dismenorreia a DP e mais recorrente ${ }^{16,17}$. Um estudo transversal que aponta dados semelhantes a esta pesquisa, onde 389 participantes foram estudadas, suas características sociodemográficas foram, a idade média foi de 21 anos, e a idade em que começaram a menstruar foi de 12 a 14 anos. Cerca de metade $(50,6 \%)$ das entrevistadas relataram ter história familiar de dismenorréia ${ }^{21}$.

Em contrapartida a outras pesquisas, em um estudo $50 \%$ a $64 \%$ das voluntárias afirmam não conhecer a respeito do histórico familiar de dismenorreia, como também dor no começa da menstruação, dor moderada e contínua, que durava 1-2 dias, e mais de dois terços da população estudada apresenta um ciclo menstrual entre 26-30 dias, com a duração do fluxo 3 a 4 dias. Nesse mesmo estudo os sintomas da DP mais relatados foram o espasmo abdominal, dor nas costas e a fraqueza ${ }^{21}$.

Uma pesquisa transversal com 130 investigou a relação da DP com o absenteísmo escolar, as participantes tinham idade entre 17 e 33 anos $(20,6 \pm 2,7$ anos), destas cento e vinte quatro voluntárias queixaram-se de DP, a intensidade da dor menstrual era moderada ou grave. Um total de 60 participantes referiu absenteísmo escolar devido à dor menstrual, essas eram as voluntárias que apresentavam dismenorreia moderada e grave ${ }^{22}$.

Segundo os domínios do questionário de McGill, nos aspectos sensitivos no GC-A do protocolo contendo o ponto simpático, obteve modificações no aspecto da dor para este domínio na avaliação intragrupos pós auriculoterapia, no entanto os grupos GE-A simpático e GE-B fígado e GC-B fígado apresentaram maior significância em relação da diminuição neste aspecto no GC-A. Na comparação intergrupos o GE-A simpático e GE-B fígado apresentaram modificações extremamente significantes neste aspecto quando comparados ao GC-A. No aspecto afetivo na interpretação intragrupos os grupos que apresentaram alterações foram GE-A simpático e GC-B fígado, nos intergrupos o resultado foi o mesmo que no aspecto sensitivo. 
No domínio avaliativo na análise intragrupos apenas o GE-A simpático e GC-B fígado apresentaram resultados pós-aplicações, na análise intergrupos não houve diferença entre os grupos para esse domínio. Os aspectos miscelâneos na avaliação intragrupos os grupos que mostraram resultados foram o GE-A simpático e GC-B fígado. Nos intergrupos o GE-A simpático e o GE-B fígado apresentaram resultados em comparação ao GC-A simpático, entretanto o GE- A simpático apresentou um resultado maior que o GE-B fígado neste aspecto.

Uma pesquisa utilizou um Protocolo de 12 atendimentos, duas vezes na semana, com duração de 5 a 10 minutos para um grupo e fez intervenção sem um protocolo no outro grupo. Durante as intervenções foram utilizados os pontos Shenmen, Tronco Cerebral, Rim, Yang do Fígado, com o objetivo de reduzir os índices de dor. O grupo com protocolo conseguiu o melhor resultado com grande redução dos níveis de dor em $36 \%$. O grupo sem protocolo conseguiu atingir um índice de redução em média de $27 \%$. Porém ambos os grupos conseguiram manter os resultados positivos ${ }^{23,24}$.

Foram montados dois protocolos neste estudo com os pontos: Simpático: Rim: Shen Men: Ovário: Útero: Endócrino, no segundo protocolo o ponto simpático foi substituído pelo ponto fígado, entretanto ambos os protocolos apresentaram significância nos resultados das intervenções.

Um estudo clínico controlado randomizado realizado com 75 profissionais de saúde, distribuiu de forma aleatória os profissionais em três grupos Grupo 1 (controle); Grupo 2 (agulha) e Grupo 3 (semente) - Para comparar as influências dos objetos utilizados durante a aplicação em relação aos escores de estresse em nível médio e alto. Os grupos de intervenção receberam oito atendimentos nos pontos Shenmen, Rim e Tronco encefálico. Os grupos semente e agulha não apresentaram resultados estatisticamente significativos para o nível médio de estresse, entretanto para o nível alto de estresse os resultados foram significativos ${ }^{25}$.

Uma pesquisa envolvendo 133 profissionais de saúde com ansiedade e dor, foram alocados em quatro grupos (controle, semente, agulha e fita adesiva) onde eram classificados quanto aos níveis de ansiedade e dor, moderado e alto. O protocolo utilizado foi à versão beta do Auricular Protocol for Pain e Anxiety contendo: ponto shen men, tranquilizante, tálamo, simpático e ponto zero. Ao final de dez atendimentos o tratamento com agulhas reduziu os níveis de ansiedade com índices de $17 \%$ de redução. Nos níveis de dor a agulha conseguiu um índice de $36 \%$ de redução. Quanto aos outros instrumentos não houve diferença entre os tempos na análise ${ }^{26}$.

Neste estudo os grupos controlados, nos quais foram utilizados pedaços de fita micropore nos pontos de acordo com o protocolo correspondente, esses também apresentaram resultados significativos em relação às modificações nas características da dor, o que pode ter ocorrido devido a um efeito placebo por indução da simulação de uma aplicação de auriculoterapia. Ambos os grupos apresentaram alguma modificação nas características da dor, no entanto os grupos experimentais apresentaram resultados mais significantes em comparação com os grupos controlados.

Segundo literaturas, o relaxamento da musculatura lisa do útero será mediante o sistema nervoso- vegetativo que será representado pelo (ponto simpático), esse ponto tem função álgica em especial para dores de origem visceral como espasmo ou cólica intestinal, biliares, renais, menstruais e dores de estômago. Os demais pontos como o (ponto rim), seu efeito analgésico pode estar relacionado à ativação de retiradas de toxinas e aumento da capacidade de oxigenação dos tecidos ${ }^{27}$.

O ponto (shen men) é também conhecido como "porta do espírito ou da mente", quando estimulado abre uma condução de subsequentes estímulos neuroquímicos capazes de liberar substâncias analgésicas. O (ponto do ovário) é muito utilizado para as disfunções ovarianas como fibromas, cistos, problemas que ocorrem na fase pré-menstrual com dores, infertilidade feminina e alguns casos de infertilidade masculina ${ }^{27}$. O (ponto útero), que é indicado nas alterações ginecológicas e obstétricas. O (ponto endócrino), regula as funções das glândulas de secreção endógenas, sendo usado em distúrbios ginecológicos, entre outros ${ }^{28}$. 
(Ponto do fígado) é localizado na concha superior, na direção do tubérculo de Darwin sempre na orelha direita. Importante no tratamento das infecções do fígado. Também é considerado um ponto hepato-protetor, auxiliando no tratamento hepático e em alguns casos no tratamento de gastrite, também é usado para intolerâncias alimentares. É um ponto auxiliar no funcionamento do intestino e encontra-se sempre na orelha direita, de destros e canhotos, é um ponto importante para trabalhar a raiva ${ }^{27}$.

Os grupos experimentais (GE) apresentaram maiores resultados em comparação com os demais grupos, entretanto se comparados entre si, o GE com o protocolo contendo os pontos, simpático, shen men, rim, ovário, útero e endócrino, (GE-A) obteve maior resultado estático do que o grupo com os pontos, fígado, shen men, rim, ovário, útero e endócrino (GE-B). O GE-A apresentou modificações nos quarto domínios do questionário de McGill na análise intragrupos e três na intergrupos, e o GE-B na intragrupos apresentou modificação em um domínio, e na intergrupos em três com resultados estáticos, porém menor que GE-A no último domínio.

\section{Conclusão}

O presente estudo obteve o delineamento nos aspectos sociodemográficos da população que possui dismenorreia primária. E em relação às influências da auriculoterapia sobre as características da dor, o método apresentou se estatisticamente significativo. Após a auriculoterapia, o grupo que mostrou maior resultado foi o experimental do protocolo contendo o ponto simpático, sendo eficaz para modificar os aspectos da dor determinando resultados benéficos para o uso do método e do protocolo.

\section{Contribuições dos autores}

Todos os autores participaram igualmente deste projeto.

\section{Conflitos de interesses}

Nenhum conflito financeiro, legal ou político envolvendo terceiros (governo, empresas e fundações privadas, etc.) foi declarado para nenhum aspecto do trabalho submetido (incluindo, mas não se limitando a subvenções e financiamentos, participação em conselho consultivo, desenho de estudo, preparação de manuscrito, análise estatística, etc.).

\section{Referências}

1. Acqua RD, Bendlin T. Dismenorreia. Femina. 2015;43(6):273276.

2. Gerzson LR, Padilha JF, Braz MM, Gasparetto A. Fisioterapia na dismenorreia primaria: revisão de literatura. Rev Dor. 2014;15(4):290-295. doi: 10.5935/1806-0013.20140063

3. Pinho LEE, Monteiro AKS, Pimenta AG, Santos ASA. Terapia manual no tratamento da dismenorreia primária: revisão sistemática. Revista pesquisa em fisioterapia. 2017;7(2):224-232. doi: $10.17267 / 2238-2704$ rpf.v7i2.1327

4. Kim J, Lee H, Kim H, Kim JY, Kim KH. Diferenças nas características da língua de pacientes com dismenorréia primária e controles sobre um ciclo menstrual normal. Evidence-based complementary and alternative medice, 2017;2017:1-9. doi: $\underline{10.1155 / 2017 / 6435702}$

5. Baviul DA, Dolatian M, Mahmoodi Z, Baghban AA. Comparasion Of Lifestyle Of Young Women With and primary dysmenorrhea. Eletronic Physician. 2016;8(3):2107-2114. doi: 10.19082/2107

6. Behbahani BM, Ansaripour L, Akbarzadeh M, Zare N, Hadianfard MJ. Comparison of the Effects of Acupressure and self - care behaviors training on intensity of primary dysmenorrhea based on McGll pain questionnaire among Shiraz University Students. Journal of Research in Medical Sciences, Iran, 2016;21:104. doi: 10.4103/1735-1995.193176

7. Jin L, Yang X, Liu P, Sun J, Chen F, Xu Z et al. Dynamic abnormalities of spontaneous brain activity in women with primary dysmenorrhea. J Pain Res. 2017;10:699-707. doi: $\underline{10.2147 /}$ JPR.S121286 
8. Kim MJ, Beak IH, Goo BO. O efeito do alinhamento lombarpélvico e da espessura do músculo abdominal na dismenorréia primária. J Phys Ther Sci. 2016;28(10):2988-2990. doi: 10.1589/ jpts.28.2988

9. Bellelis P, Dias Junior JA, Podgaec S, Gonzales M, Baracat EC, Abrão MS. Aspectos epidemiológicos e clínicos da endometriose pélvica - uma série de casos. Rev Assoc Med Bra. 2010;56(4):46771.

10. Abdelmoty HI, Youssef MA, Abdallah S, Malak KA, Hashish NM, Samir $D$ et al. Menstrual patterns and disorders among secondary school adolescents in Egypt. A cross-sectional survey. BMC Women's Health. 2015;15(70):2-6. doi: 10.1186/s12905-015-0228-8

11. Mieli MPA, Cezarino PYA, Margarido PFR, Simões R. Dismenorreia primária: tratamento. Revista da Associação Médica Brasileira. 2013;59(5):413-419.

12. Dardes RCM, Morais AS, Santos MB. Dismenorreia. Revista Brasileira de Medicina. 2011;68(12):14-20.

13. Armour M, Dahlen HG, Smith CA. More Than Needles: The Importance of Explanation and Self - Care Advice in Treating Primary Dysmenorrhea with Acupunture. Hindawi Publishing Corporation. 2016;34:416-424. doi: 10.1155/2016/3467067

14. Wang YJ, Hsu CC, Yeh ML, Lin JG. Auricular Acupressure to Improve Menstrual Pain and Menstrual Distress and Heart Rate Variability for Primary Dysmenorrhea in Youth with Stress. Hindawi Publishing Corporation. 2013;8(20):138537. doi: 10.1155/2013/138537

15. Yeh ML, Hung YL, Chen HH, Lin JG, Wang YJ. Acupressura auricular combinada com uma intervenção baseada na Internet ou sozinha para a dismenorréia primária: um estudo de controle. Evid Based Complement Alternat Med. 2013;2013:1-8. doi: $10.1155 / 2013 / 316212$

16. Frare JC, Tomadon A, Silva JR. Prevalência da dismenorreia e seu efeito na qualidade de vida entre mulheres jovens. Revista Brasileira de Ciências da Saúde. 2014;12(39):15-20.

17. Sezeremeta DC, Carvalho MSS, Vrecchi MR, Marafon RGC, Crespilho LC, Pagotto JP et al. Dismenorreia: Ocorrêia na vida de Acadêmicas da área da saúde. Revista Unopar. 2013;15(2):123126.

18. Charandabi SMA, Biglu MH, Rad KY. Efeito da homeopatia sobre a intensidade da dor e qualidade de vida dos alunos com dismenorréia primária: uma experimentação controlada aleatória. Iranian Red Crescent Medical Journal, 2016;18(9):e30902. doi: 10.5812/ircmj.30902
19. Aziato L, Dedey F, Clegg-Lamptey JN. The experience of dysmenorrhoea among Ghanaian senior high and university students: pain characteristics and effects. Reproductive Health. 2014:(58):1-8. doi: 10.1186/1742-4755-11-58

20. Armour M, Dahlen HG, Zhu X, Farquhar C, Smith CA. O papel do tempo de tratamento e do modo de estimulação no tratamento de dismenorréia primária com acupuntura: um ensaio exploratório randomizado controlado. PLos One, 2017;12(7):1-20. doi: $10.1371 /$ journal.pone.0180177

21. Gebeyehu MB, Mekuria AB, Tefera YG, Andarge DA, Debay YB, Bejiga GS et al. Prevalência, Impacto e Prática de Gestão da Dismenorréia entre estudantes da Universidade de Gondar, noroeste da Etiópia: um estudo transversal. International Journal of Reproductive Medicine, 2017;2017:1-8. doi: $10.1155 / 2017 / 3208276$

22. Nunes JMO, Rodrigues JA, Moura MSF, Batista SRC, Coutinho SKSF, Hazine FA et al. Prevalência de dismenorreia em universtárias e sua relação com absenteísmo escolar, exrcício físico e uso de medicamentos. Revi Bras Promoc Saúde. 2013;26(3):381-386. doi:10.5020/18061230.2013.p381

23. Kurebayashi LFS, Turrini RNT, Talita PBS, Marques CF, Rodrigues RTF, Charlesworth K. Auriculoterapia para redução de ansiedade e dor em profissionais de enfermagem: ensaio clínico randomizado. Rev Latino - Am. Efermagem. 2017;25:e2843. doi: 10.1590/1518-8345.1761.2843

24. Ushinohama A, Cunha BP, Costa LOP, Barela AMF, Freitas PB. Effect of a single session of ear acupuncture on pain intensity and postural control in individuals with chronic low back pain: a randomized controlled trial. Braz J Phys Ther. 2016;20(4):328-335. doi:10.1590/bjpt-rbf.2014.0158

25. Kurebayashi LFS, Silva MJP. Eficácia da auriculoterapia chinesa para redução da dor em equipes de enfermagem: ensaio clínico randomizado. Rev. Latino - Am. Efermagem.2017;22(3):371-8. doi: $\underline{10.1590 / 0104-1169.3239 .2426}$

26. Kurebayazhi LFS, Gnatta JR, Borges TP, Belisse G, Coca S, Minami A et al. Aplicabilidade da auriculoterapia com agulhas ou sementes para diminuição de estresse em profissionais de enfermagem. Rev Esc Enferm. 2012;46(1):89-95. doi: 10.1590/ $\underline{\mathrm{S} 0080-62342012000100012}$

27. Mas WDD. Auriculoterapia: Auriculomedicina na Doutrina Brasileira. Roca; 2004.

28. Mafetoni RR, Shimo AKK. Effects of auriculotherapy on labour pain: a randomized clinical trial. Rev Esc Enferm USP. 2016;50(5):726-732. doi: 10.1590/s0080-623420160000600003 